\title{
The differential sum rule for the relaxation rate in the cuprates
}

\author{
Ar. Abanov ${ }^{1}$ and Andrey V. Chubukov ${ }^{2}$ \\ ${ }^{1}$ Los Alamos National Laboratory, MS262, Los Alamos, NM 87545 and \\ ${ }^{2}$ Department of Physics, University of Wisconsin, Madison, WI 53706
}

(Dated: November 16, 2018)

\begin{abstract}
Motivated by recent experiments by Basov et al, we study the differential sum rule for the effective scattering rate $1 / \tau(\omega)$. We show that in a dirty BCS superconductor, the area under $1 / \tau(\omega)$ does not change between the normal and the superconducting states. For magnetically mediated pairing, similar result holds between $T<T_{c}$ and $T \geq T_{c}$, while in the pseudogap phase, $1 / \tau(\omega)$ is just suppressed compared to $1 / \tau(\omega)$ in the normal state. We argue that this violation of the differential sum rule is due to the fact that low-energy fermions in the pseudogap phase are still incoherent.
\end{abstract}

The analysis of the optical sum rules in condensed matter systems is a valuable tool that helps one to understand the key physics and relevant energy scales in the problem [1]. The focus of this report are recent experimental results [2] for the effective relaxation rate $\tau^{-1}(\omega)=\left(4 \pi / \omega_{p l}^{2}\right) R e[1 / \sigma(\omega)]$ where $\sigma(\omega)=\sigma_{1}(\omega)+$ $i \sigma_{2}(\omega)$ is the optical conductivity, $\omega_{p l}^{2}=4 \pi n e^{2} / m$ is the plasma frequency, and $n$ is the density of particles. The data analysis for optimally doped $\mathrm{YBa}_{2} \mathrm{Cu}_{3} \mathrm{O}_{6.95}[3]$ and $\mathrm{Tl}_{2} \mathrm{Ba}_{2} \mathrm{CuO}_{6+x}$ 相 and underdoped $\mathrm{YBa}_{2} \mathrm{Cu}_{4} \mathrm{O}_{8}$ and $\mathrm{Bi}_{2} \mathrm{Sr}_{2} \mathrm{CaCu}_{2} \mathrm{O}_{8+x}$ (2) revealed an approximate differential sum rule for $\tau^{-1}(\omega)$ between $T \geq T_{c}$ and $T<T_{c}$ : although $\int d \omega \tau^{-1}(\omega)$ does not converge, it changes very little when the system enters into the superconducting state. This differential sum rule, however, is not satisfied between the normal and the pseudogap phases $-1 / \tau(\omega)$ in the pseudogap phase appears to be just suppressed.

Quite generally, exact sum rules are related to conservation laws. The $f$-sum rule for the optical conductivity states that at a given density of particles, the total absorbing power of the solid characterized by $\sigma_{1}$ does not depend on the details of the interactions and is determined only by the total number of particles in the system [5]. The total absorption power is given by $\int_{0}^{\infty} \sigma_{1}(\omega)$ Applying the Kubo formula that relates $\sigma(\omega)$ with the full retarded current-current correlator $\Pi(\omega)$ : $\sigma(\omega)=\left(\omega_{p l}^{2} / 4 \pi\right) \Pi(\omega) /(-i \omega+\delta)$, separating the frequency integral into the integral over infinitesimally small $\omega$ and the rest and using the Kramers-Kronig relation for $\Pi(\omega)-1$ that vanishes at the highest frequencies, we obtain $\int_{0}^{\infty} \sigma_{1}(\omega)=\omega_{p l}^{2} / 8$, independent of the form of $\Pi(\omega)$.

Is there an analogous sum rule for $1 / \tau(\omega)$ ? Using $1 / \tau(\omega)=-\operatorname{Im}\left[\omega^{2} / \Pi(\omega)\right] / \omega$ and applying the KramersKronig relation, we find

$$
\int_{0}^{\infty} \frac{d \omega}{\tau(\omega)}=\frac{\pi}{2}\left[\Re \frac{\omega^{2}}{\Pi(\omega)}+C\right]=\frac{\pi}{2} C
$$

The constant $C$ again has to be chosen such that $\omega^{2} / \Pi(\omega)+C$ vanishes at $\omega \rightarrow \infty$. However, $C$ turns out to be infinite as at high frequencies $\Pi(\omega) \approx 1$, and $\omega^{2} / \Pi(\omega)$ diverges. This divergence implies that there is no conservation law associated with the relaxation rate and hence no sum rule for $1 / \tau(\omega)$.

Still, one can try to see whether one can get useful

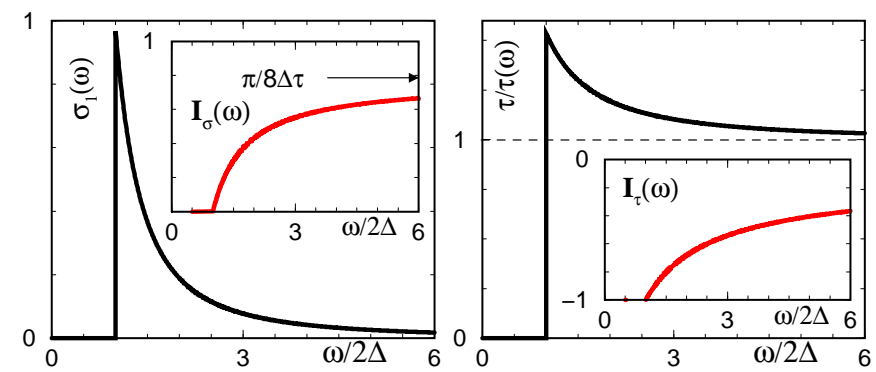

FIG. 1: The results for $\sigma_{1}(\omega)$ and $1 / \tau(\omega)$ (in arbitrary units) for a BCS superconductor, to first order in $1 / \tau \Delta$ (clean limit). In the dirty limit, the jump in the conductivity at $\omega=2 \Delta$ is much smaller. The inserts show $I_{\sigma}(\omega)=8 / \omega_{p l}^{2} \int_{0}^{\omega} d x \sigma_{1}(x)$ and $I_{\tau}(\omega)=(\tau / 2 \Delta) \int_{0}^{\omega} d x(1 / \tau(x)-1 / \tau)$. The arrow indicates the value of $I_{\sigma}(\infty)$ and. The differential sum rule for $1 / \tau(\omega)$ is satisfied if $I_{\tau}(\infty)=0$.

information by comparing $1 / \tau(\omega)$ for two different system parameters, e.g. temperatures, which do not affect the system behavior at high frequencies. Suppose momentarily that $1 / \tau\left(\omega, T_{1}\right)-1 / \tau\left(\omega, T_{2}\right)$ vanishes at high frequencies. The Kramers-Kronig relation is then fully satisfied and using (11) we immediately find that $\int d \omega\left(\tau^{-1}\left(\omega, T_{1}\right)-\tau^{-1}\left(\omega, T_{2}\right)\right)=0$, i.e., the area under $1 / \tau(\omega)$ does not change with $T$. The conservation of the total area under $1 / \tau(\omega)$ would create a valuable tool to study the evolution of the spectral weight between, e.g., the normal and the superconducting states. This new differential sum rule, however, is not associated with a conservation law and therefore is not guaranteed to be satisfied - only explicit calculations can determine whether or not the temperature dependence in $\Pi(\omega, T)$ is weak enough to ensure the convergence of $1 / \tau\left(\omega, T_{1}\right)-1 / \tau\left(\omega, T_{2}\right)$. In this communication we study under which conditions the differential sum rule for $1 / \tau(\omega)$ actually holds, and at which frequencies it is exhausted. We consider the magnetic scenario for the pairing in the cuprates, and argue that although the differential sum rule is approximately satisfied for all $T$, it is exhausted at energies comparable to the pairing gap only if there is a strong feedback effect from the pairing 
on the fermionic propagator - otherwise, the sum rule is exhausted at much larger frequencies. We associate the first regime with $T<T_{c}$, and the second one with the pseudogap phase.

To put our analysis of the spin mediated pairing into perspective we first analyze the situation in a dirty BSC $s$-wave superconductor at $T=0$, when the pairing causes a strong feedback on the fermionic propagator, and in the toy model where there is no feedback from the pairing on the fermionic self-energy.

The theory of a dirty superconductor is well developed [6, [7]. In the normal state, the inelastic scattering by impurities yields a retarded fermionic self-energy $\Sigma(\omega)=i / 2 \tau$, In a superconducting state, this self-energy is modified due to a feedback from superconductivity and takes the form $\Sigma(\omega)=(i / 2 \tau) \omega / \sqrt{\omega^{2}-\Delta^{2}}$ where $\Delta$ is the superconducting gap [6]. Substituting these forms into the current-current polarization bubble and performing the momentum integration we obtain

$$
\begin{aligned}
& \Pi(\omega)=\int_{0}^{\infty} d \Omega \frac{1}{\left(\sqrt{\Omega_{+}^{2}-\Delta^{2}}+\sqrt{\Omega_{-}^{2}-\Delta^{2}}+i / \tau\right)} \\
& \times \frac{\sqrt{\Omega_{+}^{2}-\Delta^{2}} \sqrt{\Omega_{-}^{2}-\Delta^{2}}-\Delta^{2}-\Omega_{+} \Omega_{-}}{\sqrt{\Omega_{+}^{2}-\Delta^{2}} \sqrt{\Omega_{-}^{2}-\Delta^{2}}}
\end{aligned}
$$

where $\Omega_{ \pm}=\Omega \pm \omega / 2$. In the normal state, this reduces to a conventional Drude form $\Pi(\omega)=\omega /(\omega+i / \tau)$. In the superconducting state, the frequency integral in (2) can be evaluated analytically in the clean limit $\Delta \tau \gg 1$. After lengthy but straightforward calculations we found that both $\sigma_{1}(\omega)$ and $1 / \tau(\omega)$ vanish at $\omega<2 \Delta$, while at larger frequencies

$$
\sigma_{1}(\omega)=\frac{\omega_{p l}^{2}}{4 \pi \tau \omega^{2}} E\left(\sqrt{1-\frac{4 \Delta^{2}}{\omega^{2}}}\right), \frac{1}{\tau(\omega)}=\frac{4 \pi \sigma_{1}(\omega) \omega^{2}}{\omega_{p l}^{2}}
$$

where $E(x)$ is the complete elliptic integral of the second kind [8]. At $\omega=2 \Delta+0, E=\pi / 2$ and both $\sigma_{1}(\omega)$ and $1 / \tau(\omega)$ jump to finite values. At high frequencies, $E(x \approx$ $1) \rightarrow 1, \sigma_{1}(\omega)$ vanishes as $\omega^{-2}$, and $1 / \tau(\omega)$ approaches the normal state result $\tau(\omega)=\tau$. To the same order, we also have $\Pi(0)=1-\pi /(8 \Delta \tau)$. We checked analytically that the $f$-sum rule $\left(8 / \omega_{p l}^{2}\right) \int_{+0}^{\infty} d \omega \sigma_{1}(\omega)=1-\Pi(0)$ is indeed satisfied.

Expanding $E(x)$ near $x=1$, we find that at high frequencies $\tau^{-1}(\omega)-\tau^{-1} \approx\left(2 \Delta^{2} / \omega^{2} \tau\right)(\log (2 \omega / \Delta)-0.5)$, i.e. $\int d \omega(1 / \tau(\omega)-1 / \tau)$ converges. The convergence implies that for a dirty BCS superconductor, the differential sum rule for $1 / \tau(\omega)$ is an exact one.

The plots of $\sigma_{1}(\omega)$ and $1 / \tau(\omega)$ are presented in Fig. 1 together with the results for $I_{\sigma}(\omega)=\left(8 / \omega_{p l}^{2}\right) \int_{0}^{\omega} d x \sigma_{1}(x)$ and $I_{\tau}(\omega)=(\tau / 2 \Delta) \int_{0}^{\omega} d x(1 / \tau(x)-1 / \tau)$. It follows from (3) that the sum rules for $\sigma_{1}(\omega)$ and $1 / \tau(\omega)$ should be exhausted at frequencies of the order of $\Delta$. Fig. 1 shows that this is indeed the case. We next consider
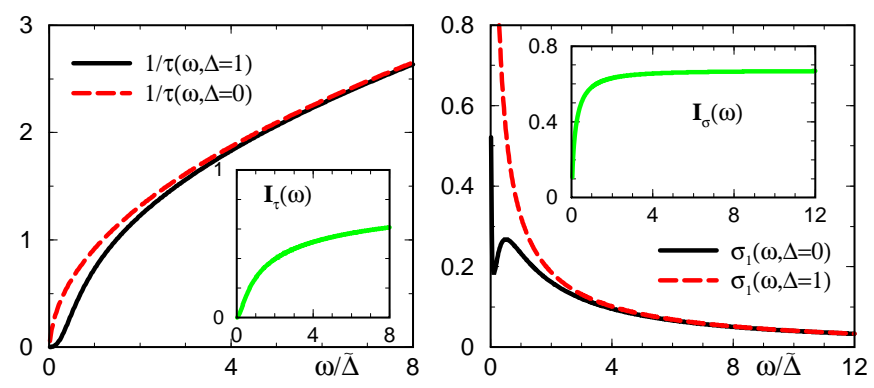

FIG. 2: The results for $1 / \tau(\omega)$ (a) and $\sigma_{1}(\omega)$ (b) for a toy model in which the pairing is not accompanied by the feedback on the electrons. The frequency is in the units of $\tilde{\Delta}$ (the peak frequency in the DOS). We used $\tilde{\Delta}=0.25 \bar{\omega}$. Observe that $1 / \tau(\omega)$ is nearly homogeneously suppressed at $\omega=O(\tilde{\Delta})$, and the differential $I_{\tau}(\omega)$ converges to zero only at $\omega \sim 10^{3} \tilde{\Delta}$ (not shown). On the other hand, $I_{\sigma}(\omega)$ converges to $I_{\sigma}(\infty) \approx 0.67$ already at $\omega \sim 4 \Delta$.

the behavior of $\sigma_{1}(\omega)$ and $1 / \tau(\omega)$ in the toy model in which we assume that the pairing does not change the fermionic self-energy. Having the cuprates in mind, we also assume, that the normal state is not a Fermi liquid, i.e., fermionic self-energy at low frequencies behaves as $\Sigma(\omega)=(i \omega)^{\alpha} \bar{\omega}^{1-\alpha}$ with $\alpha<1$,

For the frequency dependent self-energy $\Sigma(\omega)$, the current-current correlator $\Pi(\omega)$ is still given by Eq. (2), but now $\Omega$ is substituted by $\Omega+\Sigma(\Omega)$. For definiteness, we present the results for $\alpha=1 / 2$, which is the normal state quantum-critical exponent in the spinfermion theory [9], but the results are qualitatively the same for all $\alpha$ including the marginal Fermi liquid limit $\alpha \rightarrow 0$ [10]. For $\alpha=1 / 2$, the fermionic density of states $N(\omega)=\operatorname{Im}\left[\tilde{\Sigma}(\omega) /\left(\Delta^{2}-\tilde{\Sigma}^{2}(\omega)\right)^{1 / 2}\right]$ has a maximum at $\omega=\tilde{\Delta} \approx \Delta^{2} / \bar{\omega}$, that could be identified with the pseudogap. Still, $N(\omega)$ remains finite at all frequencies. The assumption that the self-energy does not change is internally consistent if indeed the anomalous normal state behavior extends to frequencies which well exceed the pairing gap, i.e., when $\bar{\omega} \gg \tilde{\Delta}$.

Evaluating $\Pi(\omega)$ and substituting it into $\sigma_{1}(\omega)$ and $1 / \tau(\omega)$ we found that in the normal state, $\sigma_{1, n}(\omega) \propto$ $(\omega \bar{\omega})^{-1 / 2}$ at $\omega \ll \bar{\omega}$ and $\sigma_{1, n} \propto\left(\bar{\omega} / \omega^{3}\right)^{1 / 2}$ at $\omega \gg \bar{\omega}$, while $1 / \tau_{n}(\omega) \propto(\omega \bar{\omega})^{1 / 2}$ in both limits. For $\Delta \neq 0$, we found that for $\omega \ll \tilde{\Delta}, \sigma_{1}(\omega) \propto(\omega \bar{\omega})^{-1 / 2}(\omega / \tilde{\Delta})^{5 / 2}$ and $1 / \tau(\omega) \propto(\omega \bar{\omega})^{1 / 2}(\omega / \tilde{\Delta})^{7 / 2}$. We see that both $\sigma_{1}(\omega)$ and $1 / \tau(\omega)$ are reduced compared to their normal state values but are still finite. At $\omega \sim \tilde{\Delta}$, both $\sigma_{1}(\omega)$ and $1 / \tau(\omega)$ become comparable to the normal state results. At larger $\tilde{\Delta} \ll \omega \ll \bar{\omega}, \sigma_{1}(\omega)=\sigma_{1, n}(\omega)-$ $1.992\left(\omega_{p l}^{2} / 4 \pi\right) \tilde{\Delta}\left(\omega^{3} \bar{\omega}\right)^{-1 / 2}$ and $1 / \tau(\omega)=1 / \tau_{n}(\omega)-$ $3.51 \tilde{\Delta}(\bar{\omega} / \omega)^{1 / 2}$. Finally, at $\omega \gg \bar{\omega}, \sigma_{1}(\omega)-\sigma_{1, n}(\omega) \propto$ $\omega^{-7 / 2} \log \omega$ and $1 / \tau(\omega)-1 / \tau_{n}(\omega) \propto \omega^{-3 / 2} \log \omega$.

We see that $\sigma_{1}(\omega)$ converges to its normal state value at 

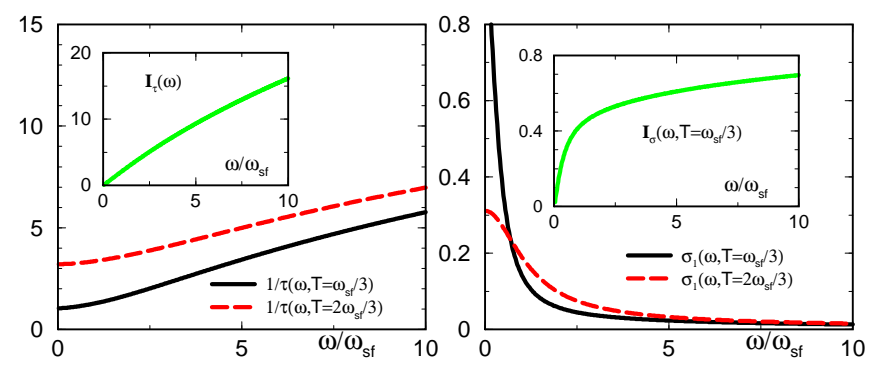

FIG. 3: The normal state results for $1 / \tau(\omega)$ (a) and $\sigma_{1}(\omega)$ (b) for the spin-fermion model for $\lambda=2$. The insets show $I_{\sigma}(\omega)$ and the differential $I_{\tau}(\omega)$ between $T=\omega_{s f} / 3$ and $T=$ $2 \omega_{s f} / 3$. The sum rule for $1 / \tau(\omega)$ is not satisfied due to weak convergence at high frequencies. $I_{\sigma}(\omega)$ flattens at $\omega \sim 10 \omega_{s f}$ but converges to the $f$-sum rule value $I_{\sigma}(\infty)=1$ only at extremely high $\omega \sim 10^{3} \omega_{s f}$ (not shown).

frequencies of order $\tilde{\Delta}$, as in a dirty BCS superconductor; the sum rule for $\sigma_{1}(\omega)$ is then exhausted at $\omega \sim \tilde{\Delta}$. This behavior is illustrated in Fig. 2a where we present the results of our numerical calculations $-I_{\sigma}(\omega)$ converges to $I_{\sigma}(\infty)=1-\Pi(0)(\approx 0.67$ for our choice of $\bar{\omega}=2 \Delta)$ already at $\Omega \sim \tilde{\Delta}$. On the other hand, $\tau^{-1}(\omega)-\tau_{n}^{-1}(\omega)$ scales as $\omega^{-1 / 2}$ between $\omega \sim \tilde{\Delta}$ and $\omega \sim \bar{\omega}$ such that at these frequencies $I_{\tau}(\omega)=\int d \omega\left(\tau^{-1}(\omega)-\tau_{n}^{-1}(\omega)\right)$ does not converge. Furthermore, at these frequencies, $1 / \tau(\omega)$ is still smaller than $1 / \tau_{n}(\omega)$. This result holds for all $\alpha<1$ as one can straightforwardly verify. Only at $\omega>\bar{\omega}$, $\tau^{-1}(\omega)$ finally becomes larger than $\tau_{n}^{-1}(\omega)$, and $I_{\tau}(\omega)$ converges. The convergence implies that the differential sum rule for $1 / \tau(\omega)$ is again exactly satisfied, however it is exhausted only at frequencies that well exceed the pseudogap. We present the numerical results for $1 / \tau(\omega)$ and $I_{\tau}(\omega)$ in Fig. 2 $\mathrm{b}$.

We now present the results for $\sigma_{1}(\omega)$ and $1 / \tau(\omega)$ for spin-fluctuation mediated $d$-wave pairing. We obtained these results by solving a set of coupled Eliashberg equations for the spin-fermion model that describes the spin fluctuation exchange at low energies 13]. We will demonstrate that at low $T$, the behavior of the conductivity and the relaxation rate resembles that in a dirty BSC superconductor, while immediately below the pairing instability the system behavior is similar to that in the toy model for the pseudogap.

The spin-fermion model is characterized by a single dimensionless coupling constant $\lambda$ and a single overall energy $\bar{\omega}$ that scales with the effective spin-fermion interaction [9]. We will also use a characteristic energy scale for the spin fluctuations $\omega_{s f}=\bar{\omega} / 4 \lambda^{2}$. A fit to the NMR, ARPES and neutron experiments yields $\lambda \sim 1-2$ near the optimal doping [9]. We refer the readers to Ref [9] for the discussion of the applicability of the model to the cuprates and the justification of the Eliashberg approach at strong spin-fermion coupling despite the formal absence of the Migdal theorem.
We begin with the normal state. In Fig. 3a we present our results for $1 / \tau(\omega)$ and $I_{\tau}(\omega)$ at various $T$. For definiteness we used $\lambda=2$. We see that $I_{\tau}(\omega)$ diverges at high frequencies, i.e., the differential sum rule is not satisfied. We checked analytically that this is caused by the $1 / \omega$ behavior of the integrand in $I_{\tau}(\omega)$.

For completeness, in Fig. 3 $3 \mathrm{~b}$ we present the results for $\sigma_{1}(\omega)$ and $I_{\sigma}(\omega)$ at various $T$. We see that $I_{\sigma}(\omega)$ flattens up at $\omega \geq 10 \omega_{s f}$, but its value is still by about $30 \%$ smaller than it should be for $\omega=\infty$. The full sum rule is exhausted only at unrealistically large $\omega \sim 10^{3} \omega_{s f}$ (Ref. [9]) where the low-energy theory is clearly inapplicable. The weak convergence of $I_{\sigma}(\omega)$ is related to the fact that over a wide frequency range $\sigma_{1}(\omega)$ is inversely proportional to $\omega$, and $I_{\sigma}(\omega)$ increases as $\log \omega$. The practical application of this result is that the plasma frequency $\omega_{p l}$ obtained by integrating the measured $\sigma_{1}(\omega)$ up to, say, $1 \mathrm{eV}$ should be about $30 \%$ less than the actual plasma frequency. This is consistent with the fact that to match the measured $\sigma_{1}(\omega \rightarrow 0)$ with the theoretical $\sigma_{1}(\omega \rightarrow 0)$ obtained using $\Sigma(\omega)$ extracted from the ARPES data, one needs the plasma frequency of about $20000 \mathrm{~cm}^{-1}$ [9, 11, while $\omega_{p l}$ extracted from the "partial" sum rule [1] is around $16000 \mathrm{~cm}^{-1}$.

We next consider what happens below the pairing instability temperature $T_{\text {ins }} \sim 0.2 \bar{\omega} 12$. Earlier Schmalian and the two of us have found [13] that at $T \leq T_{\text {ins }}$, the the fermionic self-energy remains large at the smallest $\omega$ and gradually evolves from its normal state value. This gradual behavior is qualitatively different from a dirty BCS superconductor, as in the latter the quasiparticle spectral function and the density of states instantly drop to zero at frequencies below $\Delta$ due to a feedback from the pairing [6, 7]. We found in [13] that the behavior similar to that in a BSC superconductor occurs only below $T_{c}<T_{i n s}$, and the difference between $T_{i n s}$ and $T_{c}$ increases with increasing $\lambda$. We conjectured that at $T_{c}<T<T_{\text {ins }}$, fluctuations destroy coherent superconductivity, i.e., the system is in the pseudogap regime.

In Fig. 4 we present the results for $\sigma_{1}(\omega)$ and $1 / \tau(\omega)$ for two different $\lambda$ and for three different temperatures: $T \ll T_{c}, T_{c}<T<T_{\text {ins }}$ and $T=T_{\text {ins }}$ where $\sigma_{1}(\omega)$ and $1 / \tau(\omega)$ are the same as in the normal state. We clearly see that between $T_{c}<T<T_{\text {ins }}, 1 / \tau(\omega)$ is nearly homogeneously suppressed, while at smaller $T$ it shifts from the lowest frequencies to $\omega \sim 2 \Delta(\sim 0.8 \bar{\omega})$ and develops an overshoot at $\omega \geq 2 \Delta$. The magnitude of the overshoot depends on the coupling and is larger at larger $\lambda$. We compared our results with the fermionic self-energy [13], and found that the temperature where $1 / \tau(\omega)$ develops an overshoot, agrees with the onset temperature for the feedback effects on the fermionic propagator.

For quantitative analysis of the sum rule we looked analytically into the high frequency behavior of $1 / \tau(\omega)$. We found that the temperature variation of $1 / \tau(\omega)$ at high frequencies is still strong enough to prevent the differential sum rule for $1 / \tau$. On the other hand, we see from the insert in Fig. $4 \mathrm{~b}$ that this variation can be 

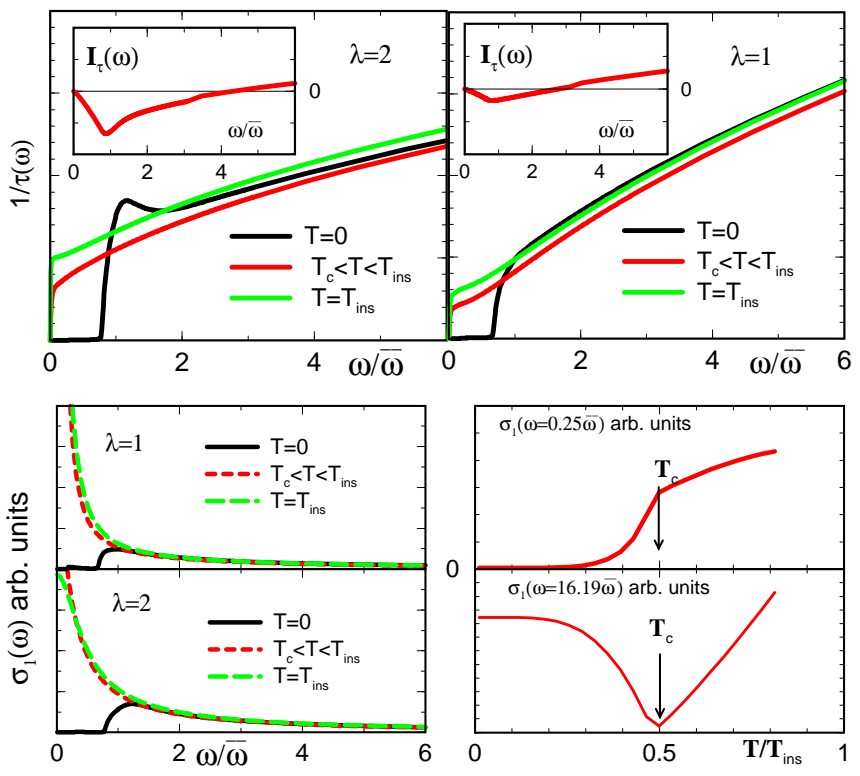

FIG. 4: The behavior of $1 / \tau(\omega)$ and $\sigma_{1}(\omega)$ in the spinfermion model below the pseudogap temperature $T_{\text {ins }}$ for $\lambda=2(\Delta \sim 0.3 \bar{\omega})$ and $\lambda=1(\Delta \sim 0.2 \bar{\omega})$. Observe that the overshoot between the spectra of $1 / \tau(\omega)$ develops only below $T_{c}$. The inserts in the upper panels show the behavior of the differential sum rule $I_{\tau}(\omega)$ between $T<T_{c}$ and $T_{c}<T<T_{i n s}$. The differential sum rule is not exact, but is approximately satisfied if $\omega \sim 3-4 \bar{\omega} \sim 10 \Delta$. The right lower panels show the behavior of $\sigma_{1}(\omega)$ vs $T$ at small and large frequencies for $\lambda=1$. Observe that the changes in $\sigma_{1}$ are confined to $T_{c}$ rather than to $T_{\text {ins }}$.

safely neglected if the frequency integration is restricted to $\omega \sim 3-4 \bar{\omega} \sim 10 \Delta$ (for optimally doped $Y B C O$, $\left.10 \Delta \sim 2500 \mathrm{~cm}^{-1}\right)$. In Fig. 和 we show the results for the differential sum rule between $T \ll T_{c}$ and $T_{c}<T<T_{\text {ins }}$. We see that the spectral weight is almost exactly conserved if the upper limit of the frequency integral is chosen close to $3-4 \bar{\omega}$. The conservation of the spectral weight between $T \ll T_{c}$ and $T \geq T_{c}$, and the apparent loss of the spectral weight between $T_{c}$ and $T_{\text {ins }}$ are the main results of recent experimental analysis [2]. We see that these results are fully reproduced in our analysis.

We also see from Fig. 1 that at small $\omega, \sigma_{1}(\omega)$ keeps increasing between $T_{i n s}$ and $T_{c}$. This indicates that the development of the pseudogap does not give rise to a suppression of the conductivity at the lowest frequencies. The latter is only reduced below $T_{c}$. The sensitivity of $\sigma_{1}(\omega=0)$ to $T_{c}$ rather than to the pseudogap temperature is consistent with the data 14]. The low frequency behavior of $\sigma_{1}(\omega=0)$ below $T_{c}$ is not captured in our theory as it is predominantly determined by impurities [15]. Finally, we found both analytically and numerically that at large $\omega, \sigma_{1}(\omega)$ also turns out to be sensitive to $T_{c}$ (see the inset in Fig. (4a). This also agrees with the data [16].

To conclude, in this paper we considered the differential sum rule for the effective scattering rate $1 / \tau(\omega)$ (the difference between the area under $1 / \tau(\omega)$ for two different temperatures). We argued that for spin-fluctuation mediated pairing, this sum rule is generally not an exact one, but is rather well satisfied below $T_{c}$ and is exhausted at frequencies compared to the pairing gap, $\Delta$. We identified this behavior with the strong feedback from the pairing on the fermionic self-energy. We found that in the pseudogap region, where feedback effects are small, the differential sum rule is (approximately) exhausted only at much larger energies, comparable to the upper limit of non-Fermi-liquid behavior in the normal state, while at $\omega=O(\Delta), 1 / \tau(\omega)$ is nearly homogeneously suppressed compared to the normal state. We argued that this behavior as well as the behavior of $\sigma_{1}(\omega)$ are consistent with the experimental data on the cuprates.

It is our pleasure to thank D. N. Basov, G. Blumberg, N. Bontemps, C. Homes, D. van der Marel, A. Millis, M. Norman, D. Pines, and J. Tu for useful conversations. We are also thankful to D. N. Basov, N. Bontemps, and J. Tu for sharing the unpublished results with us. The research was supported by NSF DMR-9979749 (A. Ch.) and by DR Project 200153 at Los Alamos National Laboratory (Ar. A.).
[1] T. Timusk and B. Statt, Rep. Prog. Phys. 62, 61 (1999).

[2] D.N. Basov, E.J. Singley and S.V. Dordevic, condmat/0103507.

[3] D.N. Basov et al. Phys. Rev. Lett. 77, 4090 (1996).

[4] A.V. Puchkov, D.N. Basov, and T. Timusk, J. Cond. Matt. Phys. 8, 10049 (1996).

[5] D. Pines and Ph. Nozières, The Theory of Quantum Liquids, Cambridge, Massachusetts, Perseus books (1999).

[6] A.A. Abrikosov A. A. and L.P. Gor'kov, Sov. Phys. JETP, 8, 1090 (1959).

[7] P.W. Anderson J. Phys. Chem. Solids, 11, 26 (1959).

[8] see also H. Westfahl and D. Morr, cond-mat/0002039.

[9] A. Abanov, A. V. Chubukov, J. Schmalian, condmat/0107421: A. Chubukov, J. Schmalian and D. Pines cond-mat/xxxx, and references therein.
[10] E. Abrahams and C. Varma, Phys. Rev. Lett. 84, 4652 (2001) and references therein.

[11] N. Shah and A.J. Millis, cond-mat/0104502.

[12] Ar. Abanov, A. V. Chubukov, and A. M. Finkel'stein, Europhys. Lett., 54 ,488-494 (2001).

[13] A. Abanov, A. V. Chubukov, and J. Schmalian, Europhys. Lett.55 369 (2001).

[14] A.F. Santander-Siro et al, cond-mat/0107161; J.J. Tu et al, cond-mat/0107349.

[15] P.A. Lee, Phys. Rev. Lett. 71, 1887 (1993); S. M. Quinlan, P. J. Hirschfeld, D. J. Scalapino, Phys. Rev. B 53, 8575 (1996).

[16] D. van der Marel, Physica C 341-348, 1531 (2000). 